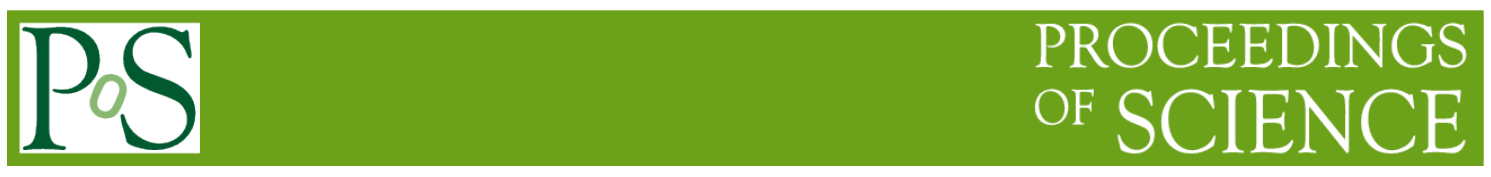

\title{
A kind of Fog Recognition Method for Video Image
}

\section{Tianwei Li}

Dept. of Navigation, Dalian Naval Academy, Dalian 116018, China;

Email: tianweilee@sina.com

\section{Fanjun Meng}

Dept. of Navigation, Dalian Naval Academy, Dalian 116018, China;

E-mail: fanjunmengg@163.com

\section{Lei Yan}

Dept. of Basic Science, Dalian Naval Academy, Dalian 116018, China

E-mail: maiyabing@163.com

\section{Jiao Guo}

Dept. of Basic Science, Dalian Naval Academy, Dalian 116018, China

E-mail: maiyabing@163.com

In order to identify the foggy weather phenomenon, this paper puts forward an automatic identification method based on the k-means clustering algorithm, which analyzes the effects on characteristics of image gathering by the foggy weather phenomenon, sets the mean value and variance extracted from the image saturation as feature parameters while the training images are classified by using k-means clustering algorithm to obtain the clustering center of different image classifications; finally the classification can be finished in the testing phase by calculating the dissimilarity between different images and the clustering center. Experimental result shows that the easy and effective method can process large-scale image data and then annotate the image categories; consequently the identification rate of foggy weather is more than $90 \%$. 


\section{Introduction}

It is well known that the modern maritime operation is multi-service joint operation. Nowadays, with the development of Chinese navy, the naval vessels navigate more and more frequently for long time to train voyage and conduct task. The traffic safety at sea should not be ignored. Among many factors which may affect the safety of maritime traffic, the weather phenomenon is one of the important factors which is uncontrollable but decisive. Navigation in foggy weather (including foul weather such as: fog, haze, rain, snow and so on) will affect positioning, navigation and collision avoiding negatively, especially be harmful to the maneuver navigation during two ships' meeting and the formation navigation. At the same time, the fog, a common weather phenomenon leading to visual barriers, does harm to the local production, living and transportation. Unfortunately, most of the weather forecast is operated manually but not automatically in weather station, so the weather information gathering is costly in terms of manpower and material. As a result, with the development of smart video-image automatic identification technology, the research on automatic identification of weather phenomenon based on video- image data attracts wide attention in recent years [1-3].

\section{Research Status}

In recent years, researchers have been studying how to identify the fog phenomenon automatically from many different angles and made many achievements. Q .Li extracts such parameters as power spectrum slope, contrast, noise, situations as features to realize the multiclassification of weather situations with Support Vector Machine(SVM), and recognizes foggy weather more than $85 \%[1]$. J .Guo concerning various kinds of low visibility weather phenomenon and based on the existing dichromatic atmospheric scattering model, proposed a modified calculation method of dichromatic model to range weather phenomenon by revising the atmospheric scattering direction, improving the calculating methods of vector angles and adding humidity parameter information [3]. X. J. Song the same scene images obtained under different weather conditions were studied. The method of multi-vertical detection was used to detect the gray value on straight blur edge, and obtained the edge spread function fitted by Sigmoid function, then got the line spread function[4]. H.J. Song proposed a traffic-video based foggy weather identification method and a fast restoration approach for traffic image blurred by foggy. Experimental results show that the proposed algorithm can not only accurately identify the current weather and improve the traffic image quality when the current weather is foggy, but also decrease the time complexity in comparison with various other algorithms and satisfy the real-time requirement [5-6]; however, the image characteristic extracted by the above methods is relatively fixed and inefficient for large-scale data processing and not specific for the foggy weather identification. In this sense, these methods can not meet the current applied demands.

In order to identify and classify the foggy weather rapidly and efficiently, the paper analyzes the video-image of foggy weather and then extracts the characteristics of the saturation and calculates the mean value and variance, finally adopts the k-means clustering algorithm for classification and identification. The basic procedure is as follows: 


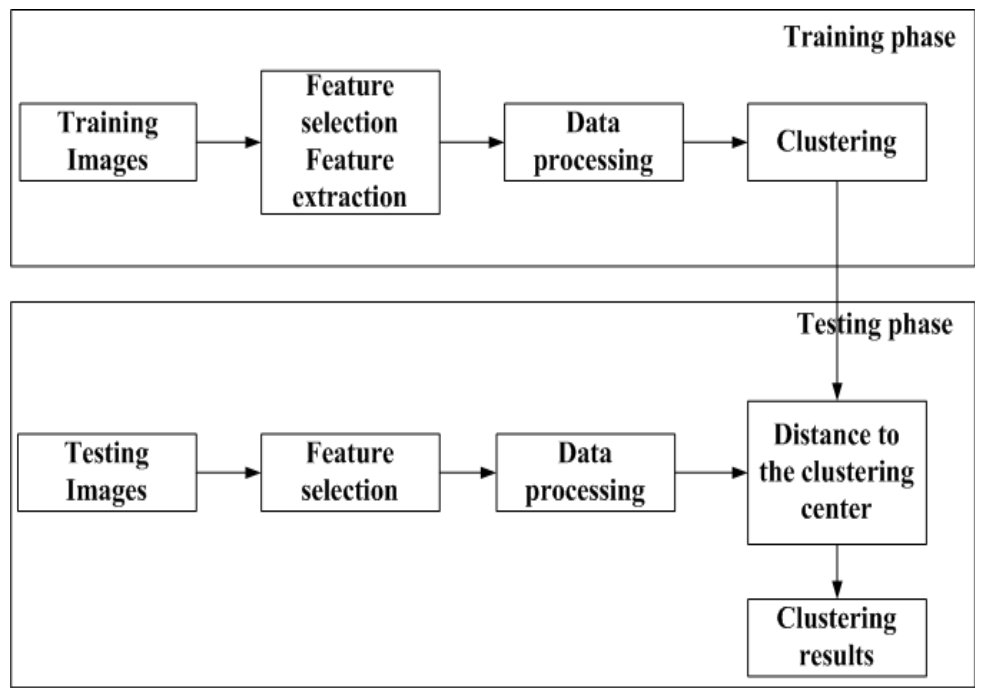

Figure1 : Procedure of Fog Identification

\section{Extracting of Image Feature}

\subsection{Effect on Image Gathering by Goggy Weather}

In the foggy weather, the atmospheric aerosol has high concentration, in visible band at the time. With the scattering effect on the light wave by aerosol, there will be a certain degree of decline in the quality of images acquired through the video imaging system[4].

After formation of the fog, the atmospheric particles may have many reflective faces, among them, some particles with large radius can keep out light and the natural light can not penetrate but will be reflected. The lights of different colors will attenuate when being reflected, so the fog becomes "white". Accordingly, the saturation of the acquired video-images will decline. On this basis, the writer regards the mean value and variance of the acquired videoimages as the feature identifying the foggy weather.

\subsection{Extracting of Foggy Weather Image Feature}

Firstly, the image is transferred from RGB mode to HSV, and then 100 images (50 foggy weather images, 50 non-foggy weather images) are randomly selected from the gathered images as training images, after that, the article extracts the saturated feature of the acquired images, figures out the mean value and variance, finally uses the k-means clustering algorithm for classification and identification.

RGB mode is the colorized mode of light. In that, R, G and B stand for red, green and blue respectively. The overlaying of such three colors leads to the other colors. HSV, a subjective color model, is used to describe the properties of color but not to break down a certain color into 3 primitive colors. HSV are the initials of hue, saturation and value. Among them, the saturation stands for the ratio of white light in colors, indicating the more pure spectrum is, the higher saturation [7] will be.

For any value of $\mathrm{R}, \mathrm{G}$ and $\mathrm{B}$ within $[0,1]$, the corresponding $\mathrm{S}$ in HSV mode can be worked out by the following Formula [7] :

$$
S=1-\frac{3}{R+G+B} \min [R, G, B]
$$


Under the condition of $\mathrm{R}+\mathrm{G}+\mathrm{B}=0$, the image will be black and $\mathrm{S}=100 \%$.

The article considering the saturation of the extracted image as the feature figures out the mean value and variance, and then forms the planar Data set.

\section{Applying K-means Clustering Algorithm in the Foggy Weather Identification}

\subsection{General Introduction of Algorithm}

The clustering describes a non-supervised statistical procedure classifying the category of physical and abstract objectives by certain algorithm. Species resulting from clustering is a category of similar data objectives. Among one species, objectives are similar, but objectives from different species are dissimilar [8]. The common clustering methods include division method, layer method, density method, gridding method and model method [8-10].

$\mathrm{K}$-means clustering is the most famous division clustering algorithm, which is widely used because of its conciseness and efficiency. Generally, given a data aggregation and the needed clustering number $\mathrm{k}$ ( $\mathrm{k}$ is designed by consumer), k-means clustering algorithm will divide the objectives into $\mathrm{k}$ number of categories by some distance functions (Euclidean distance is used in the article). During the procedure, $k$ original clustering centers are randomly selected firstly, and then the distance between every data objective and each clustering center is worked out (the Euclidean distance is figured out in the article); finally based on the calculated value, every objective will be allocated to the corresponding closest sub-category. The clustering center and cluster formed by the allocated objective represents a category. After all the objectives are allocated, it will recalculate the clustering center of cluster based on the present objectives. The procedure will be played over and over until any objective in the data category will not be assigned to different clusters or the clustering center will not change [10,11].

\subsection{Algorithm Procedure}

Step 1: pre-process and standardize the data

Extract the feature value of training image and standardize the feature value. In other words, finish the mapping from the feature value of the extracted image to the corresponding feature space, and balance the effects on distance by different values. The article maps the different feature values to the region of $[0,1]$. The mapping formula is as follows:

$$
a_{i}^{\prime}=\frac{a_{i}-\min \left(a_{i}\right)}{\max \left(a_{i}\right)-\min \left(a_{i}\right)}
$$

In the formula, $a_{i}$ is the feature value of the selected image. The article extracts the mean value and variance of image saturation to form data aggregation $\mathrm{D}$, which is used as the feature parameter.

Step 2: select the original clustering centers;

The article divides the images into 2 kinds (foggy weather and non-foggy weather), and randomly selects two elements from data category $\mathrm{D}$ as the original clustering centers of two clusters.

Step 3: calculate the dissimilarity;

The dissimilarity refer to the distance between each element to clustering center. The article respectively works out and compares the Euclidean distance between the rest elements 
and the two original clustering centers, and allocates the elements to the category of the smaller distance.

Step 4: recalculate the clustering centers

Based on the results of clustering, work out the new clustering center by using arithmetical mean value from all elements' dimension of each cluster. Based on the new clustering center, re-cluster all the elements of data category.

Step 5: circulate and iterate

Circulate the above step (Step 4) until the obtained clustering center and clustering result will not change any more.

Step 6: Send out the result [9-10].

The article extracts the image saturation feature and then figures out the saturation and variance, finally gets the clustering centers of foggy weather image and non-foggy weather image by using k-means clustering algorithm in the learning phase. In the testing phase, the tested image feature is extracted and calculated, and then the distance between the tested objective and clustering center is worked out, finally the corresponding images will be plotted out according to the smallest distance.

\section{Experimental Result and Analysis}

In order to test the effectiveness of k-means clustering algorithm on the foggy weather identification of video-image, the article uses the device of Intel(R) Core(TM)i3-2310M cpu@2.10GHZ, RAM4.00GB, and comes up with the relevant experiment in the platform of Matlab7.1. The experimental data are 1000 images, each image is manually marked with different weather phenomena such as: sunny, cloudy, rainy, foggy, dusty and so on. As the weather characteristics in the images are not obvious at night, the article randomly selects 100 daytime images (50 foggy images and 50 non-foggy images) as training samples, the sample images are as follows:
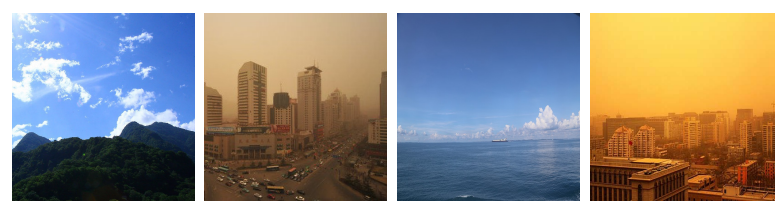

(a)Some Images of Non-foggy Weather
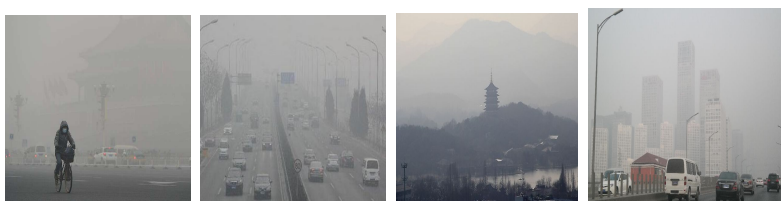

(b)Some Images of Foggy Weather

Figure 2 : Some Images of Non-foggy Weather and Foggy Weather

\subsection{Extracting of image feature}

The article analyzes 100 sample images, and then transfers the image mode from RGB space to HSV space, finally obtains the data category D by working out the mean value and variance of image saturation. The result is shown as follows: 


\begin{tabular}{|c|c|c|c|c|c|c|c|c|}
\hline & 1 & 2 & 3 & 4 & 5 & 6 & 7 & 8 \\
\hline Mean Value & 0.07 & 0.47 & 0.1 & 0.48 & 0.46 & 0.13 & 0.49 & 0.09 \\
\hline Variance & 0.01 & 0.01 & 0 & 0.01 & 0.02 & 0.02 & 0.03 & 0.03 \\
\hline
\end{tabular}

Table 1: Some Mean Values and Variances of Image Saturation

\subsection{Clustering result}

According to the procedure of k-means clustering algorithm mentioned in Section 4.2, the article randomly selects two elements from category D as two clustering centers firstlyI. In order to make calculation more persuadable, we select two clustering images before classification including some marked foggy weather images. The following figure is the distribution of image feature data before grouping.

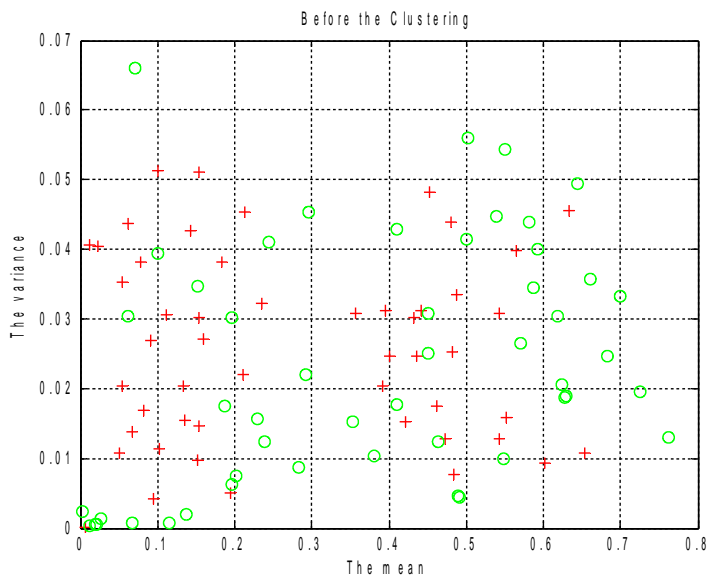

Figure 3: Data before Clustering

Denotation "+" and "O" stands for two clustering data before classifying respectively.

After clustering-analysis of the sample image data, the result is as follows:

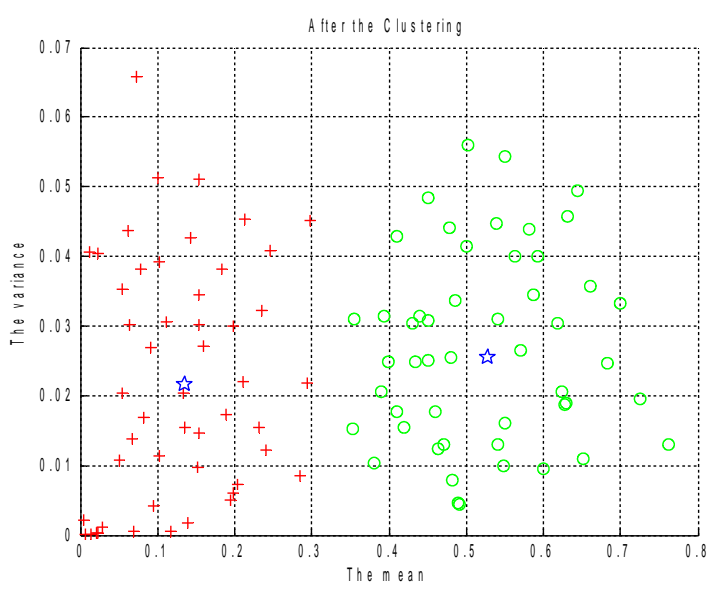

Figure 4: Data after Clustering

The denotation "+" stands for the first group of image after k-means clustering analysis (foggy weather image), the denotation "O" stands for the second group of image after k-means clustering analysis (non-foggy weather image). The denotation "s 3 " stands for the two calculated clustering centers: $[0.1313,0.0215],[0.5365,0.0256]$. 
In the testing phase, the article selects 100 representative images from the image category to work out the Euclidean distance between the tested objective and clustering center, and then carry out classification according to the smallest distance. The experimental result shows that the algorithm can effectively identify the foggy weather of video-image data, and the identification accuracy can reach $90 \%$.

Finally, this paper conducts many experiments and compares other algorithms (such as FCM algorithms, KNN algorithm and the algorithms listed in Research Status). The result shows that the speed of the method in the paper is faster and the outcome is more accurate; however, it is hard to differentiate the image of rainy weather image from that of foggy weather .

\section{Conclusion}

The article realizes the foggy weather identification in video-image data by adopting kmeans clustering algorithm. The experiment proves that the algorithm can live up to our expectations because easy algorithm can process large-scale data efficiently. Experimental result shows that the identification accuracy can reach $90 \%$; however, the article is only confined to differentiate the foggy weather from the non-foggy weather. For the more complicated weather identification, the more sophisticated algorithm is needed $[11,12]$. In the future study, we will put great emphasis to the following aspects: differentiating the rainy weather from the foggy weather in video-images; sorting out the light fog, mild fog and heavy fog in the foggy weather; and classify other weather phenomena.

\section{References}

[1] Q .Li, Y .Fan, J .Zhang, B.Q. Li. Method of weather recognition based on decision-tree-based SVM [J].Journal of Computer Applications.2011, 11(06):1624-1627. (In Chinese)

[2] M Roser, F Moosmann. Classification of weather situations on single color images[C]. IEEE Intelligent Vehicles Symposium .Eindhoven: IEEE Computer Society Press, 2008: 798-803.

[3] J .Guo, L. Yang, K. J. Wu, S. Q. Ma, S.Z. Hu. Recognition of Weather Phenomenon with Low Visibility on Basis of Improved Dichromatic Atmospheric Scattering Model [J].Meteorological Science and Technology.2013, 21(02):236-241. (In Chinese)

[4] X. J. Song, L. Yang.The Weather Phenomena Identification Based on Image Degradation Model [J].JOURNAL OF CHENGDU UNIVERSITY OF INFORMATION TECHNOLOGY.2011,22 (02):132-136. (In Chinese)

[5] H.J. Song, Y.Z. Chen, Y.Y. Gao. Research on the Method for Fog Detection and Removal Based on Traffic Video [J]. Control Engineering of China.2013, 31(06):1156-1160. (In Chinese)

[6]S G Narasimham, S K Nayar. Contrast restoration of weather degraded images [C]. IEEE Transactions on Pattern Analysis and Machine Intelligence, 2003, 25 (6): 713-724. 
[7] X.P. Qin, X.Z. Zheng. MATLAB Image Processing [M].BEIJING: PUBLISHING HOUSE OF ELECTRONICS INDUSTRUY, 2011: 115-120. (In Chinese)

[8] K .Luo, B.Y. Cai, Y.F. Wu. The Research of Clustering in Data Mining [J]. Computer Engineering and Applications . 2003, 26(20):182-184. (In Chinese)

[9] Macqueen. Some methods for classification and analysis of multivariate observations [C] .

Proc.5th Berkeley Symp. Math.Statist.1967,(1):283-297.

[10] Y.F. Xu, C.M. Chen, Y.Q. Xu. An Improved Clustering Algorithm for K-means [J].Computer Applications and Software.2008,25(3):275-277 (In Chinese)

[11]J.Y. Xie, S. Jiang, C.X. Wang .An Improved Global K-means Clustering Algorithm[J].Journal of Shanxi Normal University(Natural Science Edition).2010,23(02):18-22. (In Chinese)

[12]G.L. Xu, X.T. Wang, X, G. Xu. Improved Bi-Dimensional Empirical Mode Decomposition based on 2D Assisted Signals Analysis and Application [J]. IET Image Processing, 2011,5 (3):205-221. 